Research Article

\title{
Prevalence of Leukemia and Associated Factors among Patients with Abnormal Hematological Parameters in Jimma Medical Center, Southwest Ethiopia: A Cross-Sectional Study
}

\author{
Woldeteklehaymanot Kassahun (iD, ${ }^{1}$ Girum Tesfaye, ${ }^{2}$ Lealem Gedefaw Bimerew, ${ }^{2}$ \\ Diriba Fufa, ${ }^{3}$ Wondimagen Adissu, ${ }^{2}$ and Tilahun Yemane ${ }^{2}$ \\ ${ }^{1}$ Department of Medical Laboratory Sciences, College of Medicine and Health Sciences, Arbaminch University, \\ Arbaminch, Ethiopia \\ ${ }^{2}$ School of Medical Laboratory Sciences, Faculty of Health Sciences, Institute of Health, Jimma University, Jimma, Ethiopia \\ ${ }^{3}$ Department of Pediatrics, Faculty of Medical Science, Institute of Health, Jimma University, Jimma Medical Center, \\ Jimma, Ethiopia \\ Correspondence should be addressed to Woldeteklehaymanot Kassahun; teklu1142@gmail.com
}

Received 11 April 2020; Revised 26 August 2020; Accepted 7 November 2020; Published 19 November 2020

Academic Editor: Aldo M. Roccaro

Copyright (C 2020 Woldeteklehaymanot Kassahun et al. This is an open access article distributed under the Creative Commons Attribution License, which permits unrestricted use, distribution, and reproduction in any medium, provided the original work is properly cited.

\begin{abstract}
Introduction. Leukemia is a heterogeneous group of hematological disorder which comprise several diverse and biologically distinct subgroups. Leukemia represents the 11th and 10th most frequent cause of cancer morbidity and mortality worldwide, respectively. Adequate data regarding the prevalence of leukemia are lacking in Ethiopia, particularly in the study area. This study is aimed to determine the prevalence of leukemia and associated factors among patients who have abnormal hematological parameters in Jimma Medical Center. Methodology. A facility-based cross-sectional study was conducted involving 332 patients who have abnormal hematological parameters. Complete blood count from venous blood was made with Sysmex autohematology analyzer (Sysmex XS-500i and XT-1800; Kobe, Japan). Peripheral blood morphology and bone marrow aspirate examination were done for each patient. Descriptive statistics for the prevalence of leukemia and multinomial logistic regression analysis to assess associated factors were executed with IBM SPSS version 25. Results. The prevalence of leukemia was 9.3\%, while acute myeloid leukemia, Acute Lymphoid Leukemia, Chronic Myeloid Leukemia, Chronic Lymphoid Leukemia, Myelodysplastic Syndrome, and undifferentiated leukemia comprises $3.6 \%, 2.7 \%, 1.8 \%, 0.6 \%$, and $0.3 \%$, respectively. Older Age $(p=0.019)$, being male $(p=0.047)$, being anemic $(p=0.03)$, and rural residency of a patient $(p=0.044)$ were significantly associated with having acute myeloid leukemia. Conclusion. The prevalence of leukemia among patients who have abnormal hematological parameters in Jimma Medical Center is significant which needs further comprehensive investigations of the associated factors and predictors with more up to date diagnostic methods.
\end{abstract}

\section{Introduction}

Leukemia is a heterogeneous group of hematological malignancies that comprise several diverse and biologically distinct subgroups. It is a clonal neoplasm of hematopoietic cells resulting from arrays of factors leading to somatic mutations in pluripotent stem cells and progenitor cells. Mutated neoplastic cells behave like a hematopoietic stem cell in that it can self-replicate, differentiate, and feed progenitor cells into the various hematopoietic lineages. These leukemic, unipotently stem cells can undergo varying degrees of maturation to phenocopies of mature blood cells $[1,2]$.

The exact cause of leukemia is not yet clear. However, several factors, mainly hereditary inheritance, genetic mutations, epigenetic lesion, ionic radiation, chemical and other occupational exposures, therapeutic drugs, smoking, and some viral agents, have been implicated in developing leukemia [3-8]. 
Two types of classification systems are commonly used for leukemia: The French-American-British (FAB) classification system which is based on morphology and cytochemical staining to define specific types of leukemia and the World Health Organization (WHO) which reviews classification information, cellular morphology, cytochemistry, immunophenotyping, cytogenetics, and clinical features to define and categorize clinically significant disease entities $[9,10]$.

In the 1970s, a group of French, American, and British leukemia experts divided myeloid leukemia into acute myeloid leukemia (AML-with subtypes, M0 through M7), Chronic Myeloid leukemia (CML), and myelodysplastic syndromes (MDS) based on the type of cell, leukemia develops from and how mature the cells are. This was based mainly on how the leukemia cells looked under the microscope after routine staining and some cytochemical characteristics $[9,11,12]$.

Lymphoid malignancies represent a heterogeneous group of disorders divided into four categories based on the maturity of the neoplastic cells and the distribution of disease as acute lymphoblastic leukemia (ALL), chronic lymphoblastic leukemia (CLL), malignant lymphoma and plasma cell neoplasms, and hairy cell leukemia [13].

Leukemia is the $8^{\text {th }}$ to $12^{\text {th }}$ most common cancer disease in the globe. It affects all nations and peoples of the world without discrimination based on their sociodemographic background, even though it has a greater risk in urbanized populations [14].

Leukemia caused about 265,471 deaths worldwide in 2012, where 24,400 were in Europe, 1,840 in Australia, 21,121 in Africa (by 2012), and 3,230 deaths in Ethiopia by 2014 [15-17].

Leukemia affected 200,676 males and 151,289 females (with an ASR 5.6 and 3.6, respectively) globally. In Europe, there were 46,449 males and 35,880 female patients diagnosed with leukemia in 2012. About 233,451 populations were diagnosed with leukemia in Australia, Asia, and the USA by 2017. In contrast, it has an incidence of 23,928 cases (ASR 3.0 per 100,000) in Africa by 2012 [15-17].

Recently in 2015, a population-based cancer registry data analysis and estimation is done in Ethiopia revealed that about 1,386 and 1,886 leukemia cases had been diagnosed with an age-adjusted incidence rate of 2.8 and 3.8 in men and women, respectively. It also stated that leukemia was the most common cancer among children (accounting for $29 \%$ of 3,707 pediatric cancer cases and acute leukemia accounted for $89 \%$ (of which $91 \%$ was acute lymphocytic leukemia and 9\% was acute myeloid leukemia)) of all the leukemia cases in children [18].

The impact of leukemia in developing countries is enormous due to premature death of children, loss of parents, loss of productivity due to a disability, and high medical cost that affects the socioeconomic and health welfare of the population [19-21]. While leukemia is dealt with very well in the developed world, there is little evidence on the current status of the disease in Ethiopia in general and in the study area in particular. Therefore, this study aims to determine the prevalence of leukemia and associated factors among patients who have abnormal hematological parameters in Jimma Medical Center.

\section{Methodology}

2.1. Study Setting. A facility-based cross-sectional study was conducted in Jimma Medical Center (JMC), Jimma, Southwest Ethiopia. The study was conducted from January 1, 2019, to April 30, 2019. A total of 322 patients who have abnormal hematological parameters (out of the total patients who visited JMC in the study period) and who fulfill the inclusion criteria to participate were involved in the study consecutively.

2.2. Data Collection and Analysis. Study subjects were recruited with a consecutive sampling technique. Sociodemographic information was collected by using a structured, pretested questionnaire. A $4 \mathrm{ml}$ venous blood sample was obtained from each patient for complete blood count (CBC) analysis, peripheral morphology assessment, and cytochemical study.

Laboratory diagnostic procedures were done according to the standard operation procedures (SOPs) of each test mentioned (complete Blood Count-CBC, Peripheral Morphology examination, and bone marrow aspiration) as per the diagnostic material provider. $\mathrm{CBC}$ automation was done on Sysmex XS-500i and XT-1800 CBC analyzers (Sysmex Corporation Kobe, Japan).

For patients' who have below or above the reference interval of RBC count, hemoglobin concentration, WBC count, and platelet count [22], peripheral morphology was examined with Wright's stain and Sudan Black B (SBB) stain.

After peripheral morphology examination, for those patients suspected for leukemia (patients with peripheral blood morphology pictures showing WBC abnormalities including immature cells, morphological defect, and/or abnormal reaction to $\mathrm{SBB}$ with abnormalities on RBCs (abnormal hemoglobin concentration, size and/or shape) and platelets morphology) bone marrow aspirate examination was performed with Wright's stain and Sudan black B stain to confirm and categorize the specific group of leukemia.

Sudan Black B stain was used to differentiate myeloid cell lineages from the lymphoid in acute leukemia patients by examining the presence of black granular staining granules in the myeloid cell lineage while lymphoid cells do not stain.

2.3. Quality Assurance. The questionnaire was pretested before collecting patient information. The quality of data from $\mathrm{CBC}$ automation was assured by running quality control blood samples daily, whereas the interpretation of the microscopic examination of Wright's stain and Sudan Black B stain was cross-checked by using a control sample and confirmed by a senior hematologist. All laboratory procedures were done as per manufacturers' instructions.

2.4. Statistical Analysis. Data were entered in Epidata version 4.4 and analyzed by IBM SPSS version 25. Descriptive statistical analysis was made, including sums, proportions, 
percentage, mean, median, and standard deviations. Multinomial logistic regression analysis was executed to assess the association between variables. Data outputs were also presented with charts, tables, and figures accordingly.

2.5. Ethical Approval and Consent to Participate. Ethical clearance was obtained from the Jimma University Institute of Health, Institutional Review Board (IRB).

A letter of support was obtained from the Health Research and Postgraduate director's office and School of Medical Laboratory Sciences. Permission to conduct the study was obtained from JMC Clinical Director's office. Written informed consent was obtained from each patient before data collection.

\section{Results}

3.1. Sociodemographic Characteristics. In this study, a total of 332 study participants were included. Among these, 180 (54.2\%) were female. Most of the study participants, 179 (53.91\%), were within the age range of 15-49 years with a mean age of $23 \pm 16.5$ years. Of the study participants, 188 (57.0\%) were urban residents. On the other hand, from a total of 332 study patients who have abnormal hematological parameters, $232(69.88 \%)$ have provided their occupational status, whereas $100(30.12 \%)$ did not comply with any occupation. Of these patients, government employees and students have the highest figure $(66,19.88 \%$ each), while the least frequent were private workers (39, $11.75 \%$ ) (Table 1).

3.2. Prevalence of Leukemia. From the 332 patients studied, 31 (9.3\%) had leukemia annotated with peripheral morphology with Sudan Black B cytochemical stains. On the other hand, the rest of the patients had leukocytosis $(60.9 \%$ \& 56.5\%, 49.3\% \& 57.1\%, 57.1\% \& 50\% among male and female study participants aged $0-14,15-49$, and over 50 years of age, respectively), leukocytopenia (21.9\% \& $14.5 \%$, $8 \%$ \& $10.5 \%$ among male and female study participants aged $0-14$ and $15-49$ years of age), anemia ( $57.8 \% \& 54.8 \%, 73.3 \%$ \& $72.4 \%, 71.4 \%$ \& $75.0 \%$ among male and female study participants aged $0-14,15-49$, and over 50 years of age, respectively) and thrombocytopenia (50\% \& 74.2\%, 62.7\% \&55.2\%, 50.0\% \& 41.7\% among male and female study participants aged $0-14,15-49$, and over 50 years of age, respectively).

The highest frequency of leukemia was seen in males $51.6 \%(n=16)$. The proportion of acute leukemia was $70.96 \%$, while Chronic Leukemia and Myelodysplastic Syndrome account for $25.8 \%$ and $3.2 \%$, respectively. The most frequent type was acute myeloid leukemia (AML) with a prevalence of $3.6 \%(n=12)$. Compared to Urban residents, the highest prevalence of leukemia was seen in patients who came from a rural area which is $6.9 \%(n=23)$.

By leukemia subtype, from the total of 332 study patients who have abnormal hematological parameters, $3.6 \%$ had AML, 2.7\% had ALL, 1.8\% had CML while CLL, MDS, and undifferentiated leukemia had a prevalence of $0.6 \%, 0.3 \%$, and $0.3 \%$, respectively (Figure 1 ).

3.3. Hematological Parameters between Types of Leukemia. The distribution of some hematological parameters with respect to leukemia types with reference values of WBC count $\left(3.31-11.62 \times 10^{9} / \mathrm{L}\right)$, platelet counts $\left(145.5-444.5 \times 10^{9} / \mathrm{L}\right)$, neutrophil count $\left(1.01-7.22 \times 10^{9} / \mathrm{L}\right)$, eosinophil count $\left(0.05-1.21 \times 10^{9} / \mathrm{L}\right)$, and basophil count $\left(0.01-0.05 \times 10^{9} / \mathrm{L}\right)$ was analyzed and their proportions were calculated. In Acute leukemia, neutropenia $(60.0 \%)$ and thrombocytopenia (76.2\%) were found, while in chronic leukemia, neutrophilia (71.4\%), basophil leukocytosis (100.0\%), and eosinophilia $(85.7 \%)$ were recorded. Leukocytosis was observed in all types of leukemia (Table 2).

3.4. Associated Factors of Leukemia. Multinomial logistic regression was carried out to find the statistical association of leukemia and related factors. By using a 95\% confidence interval and $p<0.05$ it was found that being older age $(p<0.019)$, being male in sex $(p=0.047)$, being anemic $(p=0.03)$, and rural residency $(p=0.044)$ of the patient were significantly associated with having leukemia. With this model, $54.1 \%$ of the variables which affected the probability of having acute myeloid leukemia were explained (Table 3 ).

\section{Discussions}

Knowledge of the prevalence of leukemia in a population may predict etiologic hypotheses for disease control and help for effectual management of leukemia and other hematological malignancies. In developing countries, especially in Africa, there is little information on the burden and the pattern of hematological malignancies, specifically, leukemia. In this study, the overall prevalence of leukemia was 9.3\% (95\% CI $=8.8 \%-9.8 \%)$, which is significantly higher than a report of Australian $(p=3.7 \%)$ [23]. As per the GLOBOCAN (Global Cancer Incidence, Mortality, and Prevalence; a project of the International Agency for Research on Cancer (IARC)) estimate for 2018, the prevalence of leukemia in Ethiopia was estimated as $7.4 \%$, while the result of this study shows a relatively higher figure at $95 \%$ confidence interval [14]. These differences may arise due to differences in source population and demographic structure.

The prevalence of leukemia is thought to be different across sex because of biological factors. There were 332 patients in this study where the male to female ratio is almost equal, even though the female slightly dominated $(1: 1.06)$, but concerning leukemia preponderance, males slightly overweight $(1.06: 1)$ even though it is not statistically significant $(p=0.147)$. This figure is in line with study from Australia, Ireland, Canada, and Ethiopia [15, 18, 24, 25].

Leukemias may present at all ages, from the newborn to the very old, but different forms have different age distributions [26]. The mean age of this study was $23 \pm 16.5$ years with a range of 0 to 73 years of age. Comparing within each age group, the highest proportion of leukemia (35.5\%) was seen among patients older than 50 years of age while there 
TABLE 1: Sociodemographic characteristics of patients who have abnormal hematological parameters in Jimma Medical Center from January 1, 2019, to April 30, 2019.

\begin{tabular}{|c|c|c|c|c|}
\hline \multirow{2}{*}{ Characteristics } & & \multicolumn{2}{|c|}{ Sex of the patient } & \multirow{2}{*}{ Total $(N(\%))$} \\
\hline & & Male $(N(\%))$ & Female $(N(\%))$ & \\
\hline \multirow{3}{*}{ Age category } & $0-14$ & $63(49.6)$ & $64(50.4)$ & $127(38.25)$ \\
\hline & $15-49$ & $75(41.9)$ & $104(58.1)$ & $179(53.91)$ \\
\hline & $50+$ & $14(53.8)$ & $12(46.2)$ & $26(7.83)$ \\
\hline \multirow{2}{*}{ Residency } & Urban & $83(44.1)$ & $105(55.9)$ & $188(57)$ \\
\hline & Rural & $69(47.9)$ & $75(52.1)$ & $144(43.37)$ \\
\hline \multirow{5}{*}{ Occupation } & Student & $32(48.5)$ & $34(51.5)$ & $66(19.88)$ \\
\hline & Farmer & $30(49.2)$ & $31(50.8)$ & $61(18.37)$ \\
\hline & Gov't employee & $25(37.9)$ & $41(62.1)$ & $66(19.88)$ \\
\hline & Private & $22(56.4)$ & $17(43.6)$ & $39(11.75)$ \\
\hline & Unexplained & $43(43.0)$ & $57(57.0)$ & $100(30.12)$ \\
\hline Total $(N(\%))$ & & $152(45.8)$ & $180(54.2)$ & $332(100.0)$ \\
\hline
\end{tabular}

$N=$ number and $\%=$ percentage.

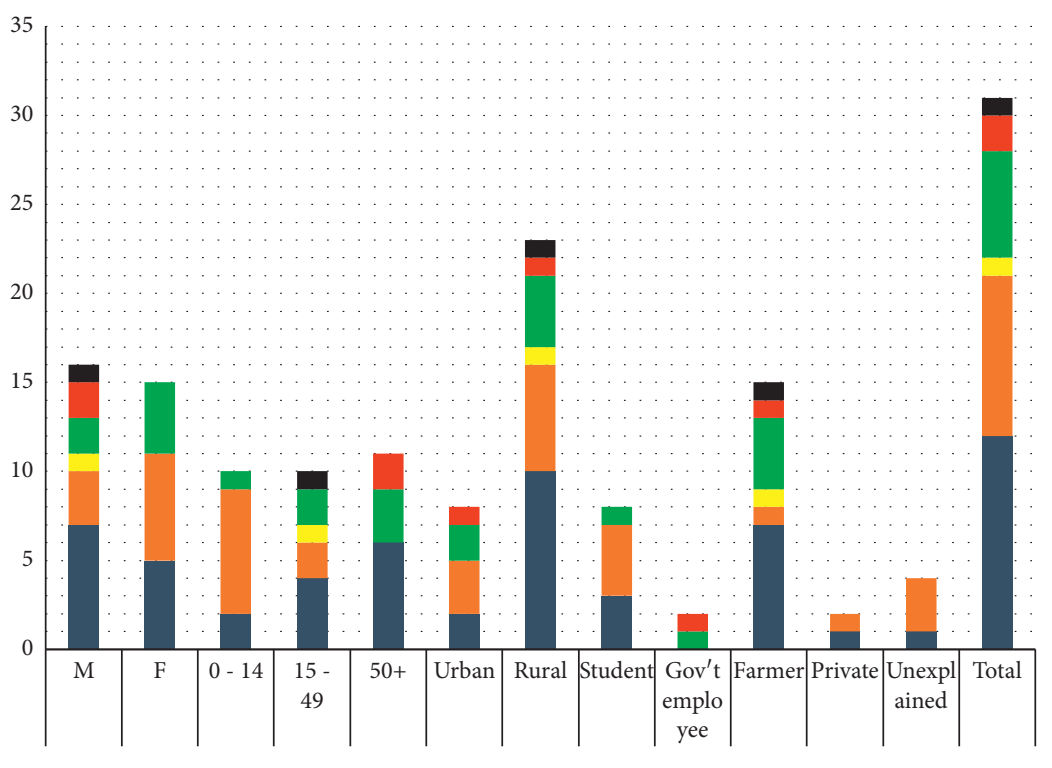

$\begin{array}{llllllllllllll}\text { - MDS } & 1 & 0 & 0 & 1 & 0 & 0 & 1 & 0 & 0 & 1 & 0 & 0 & 1 \\ \text { - CLL } & 2 & 0 & 0 & 0 & 2 & 1 & 1 & 0 & 1 & 1 & 0 & 0 & 2 \\ \text { - CML } & 2 & 4 & 1 & 2 & 3 & 2 & 4 & 1 & 1 & 4 & 0 & 0 & 6 \\ \text { Undifferentiated } & 1 & 0 & 0 & 1 & 0 & 0 & 1 & 0 & 0 & 1 & 0 & 0 & 1 \\ \text { - ALL } & 3 & 6 & 7 & 2 & 0 & 3 & 6 & 4 & 0 & 1 & 1 & 3 & 9 \\ \text { - AML } & 7 & 5 & 2 & 4 & 6 & 2 & 10 & 3 & 0 & 7 & 1 & 1 & 12\end{array}$

Figure 1: Prevalence of leukemia among patients who have abnormal hematological parameters in Jimma Medical Center from January 1, 2019, to April 30, 2019.

was a similar proportion between age groups 0-14 and 15-49 (32.3\% each) $(p<0.0001)$. This high preponderance of older adults for leukemia may be mainly due to advancement of age where many environmental exposures to carcinogens, irradiations, and malignant mutations due to clonal expansion occur more frequently [27, 28].

Environmental factors, even though not well articulated, influence the chance of developing leukemia. In this study, the highest proportion of $74.2 \%$ out of the total of 31 patients diagnosed with leukemia was observed among rural residents $(p<0.0003)$. In Ethiopia, rural residents' lifestyle is based on agricultural activities such as farming and plantations agriculture (especially coffee plantation is the major practice around the study area). Thus, this may lead to the frequent use of chemicals such as pesticides, herbicides, and fertilizers for agricultural activities which will result in genetic mutations conferring leukemia [29].

Similarly, regarding the occupation distribution of patients who have leukemia, the highest proportion of $55.6 \%$ was recorded in patients who were farmers $(p<0.001)$. This result corresponds with the case that they were from rural residency because they have varieties of exposure, which can lead to leukemogenesis [30]. The second-highest proportion 
TABLE 2: Distribution of hematological parameters counts for leukemia type of patients who have abnormal hematological parameters in JMC from January 1, 2019, to April 30, 2019.

\begin{tabular}{|c|c|c|c|c|c|}
\hline \multirow{2}{*}{$\begin{array}{l}\text { Hematological } \\
\text { parameters }\end{array}$} & & \multicolumn{4}{|c|}{ Leukemia types } \\
\hline & & $\begin{array}{l}\text { Acute leukemia } \\
\qquad(N(\%))\end{array}$ & $\begin{array}{l}\text { Chronic leukemia } \\
(N(\%))\end{array}$ & $\begin{array}{c}\text { Myelodysplastic syndrome } \\
(N(\%))\end{array}$ & $\begin{array}{c}\text { Nonmalignant } \\
(N(\%))\end{array}$ \\
\hline \multirow{3}{*}{ White blood cell count } & Leucopenia & $4(19.0)$ & 0 & $1(50.0)$ & $37(12.3)$ \\
\hline & Normal & $3(14.3)$ & 0 & 0 & $104(34.6)$ \\
\hline & Leukocytosis & $14(66.7)$ & $8(100.0)$ & $1(50.0)$ & $160(53.2)$ \\
\hline \multirow{3}{*}{ Platelet count } & Thrombocytopenia & $16(76.2)$ & $2(25.0)$ & $2(100.0)$ & $153(50.8)$ \\
\hline & Normal & $5(23.8)$ & $4(50.0)$ & 0 & $118(39.2)$ \\
\hline & Thrombocytosis & 0 & $2(25.0)$ & 0 & $30(10.0)$ \\
\hline \multirow{3}{*}{ Neutrophil count } & Neutropenia & $12(60.0)$ & $2(28.6)$ & $2(100.0)$ & $73(27.5)$ \\
\hline & Normal & $2(10.2)$ & 0 & 0 & $100(37.7)$ \\
\hline & Neutrophilia & $6(30.0)$ & $5(71.4)$ & 0 & $92(34.7)$ \\
\hline \multirow{3}{*}{ Basophil count } & Low & $7(38.9)$ & 0 & $1(50.0)$ & $78(28.7)$ \\
\hline & Normal & $4(22.2)$ & 0 & 0 & $118(43.4)$ \\
\hline & High & $7(38.9)$ & $6(100.0)$ & $1(50.0)$ & $76(27.9)$ \\
\hline \multirow{3}{*}{ Eosinophil count } & Low & $11(64.7)$ & 0 & $1(50.0)$ & $111(39.6)$ \\
\hline & Normal & $5(29.4)$ & $1(14.3)$ & $1(50.0)$ & $168(60.0)$ \\
\hline & High & $1(5.9)$ & $6(85.7)$ & 0 & $1(0.4)$ \\
\hline
\end{tabular}

$N=$ number and $\%=$ percentage.

TABLE 3: Multinominal regression model of AML for patients who have abnormal hematological parameters in Jimma Medical Center from January 1, 2019, to April 30, 2019.

\begin{tabular}{|c|c|c|c|c|c|}
\hline \multirow{2}{*}{ Parameters } & \multirow{2}{*}{$B$} & \multirow{2}{*}{ Sig. } & \multirow{2}{*}{$\operatorname{Exp}(B)$} & \multicolumn{2}{|c|}{$95 \%$ CI for $\exp (B)$} \\
\hline & & & & LB & UB \\
\hline Intercept & -45.902 & 0.034 & & & \\
\hline $\begin{array}{l}\text { Age of the } \\
\text { patient }\end{array}$ & 0.826 & 0.019 & 2.285 & 1.144 & 4.565 \\
\hline WBC $\left(\times 10^{9} / \mathrm{L}\right)$ & 0.148 & 0.153 & 1.159 & 0.946 & 1.420 \\
\hline $\mathrm{RBC}\left(\times 10^{12} / \mathrm{L}\right)$ & -11.637 & 0.030 & $8.83 E-6$ & $2.39 E-10$ & 0.326 \\
\hline $\mathrm{Hgb}(\mathrm{g} / \mathrm{dL})$ & 2.037 & 0.131 & 7.670 & 0.545 & 107.930 \\
\hline $\begin{array}{l}\text { Platelet } \\
\left(\times 10^{9} / \mathrm{L}\right)\end{array}$ & 0.017 & 0.147 & 1.017 & 0.994 & 1.040 \\
\hline Male & 9.052 & 0.047 & $8.5 \times 10^{3}$ & 1.146 & $63.6 \times 10^{6}$ \\
\hline Female & $0^{\mathrm{a}}$ & - & - & - & - \\
\hline $\begin{array}{l}\text { Urban } \\
\text { resident }\end{array}$ & -23.729 & 0.044 & $4.9 E-11$ & $4.59 \mathrm{E}-21$ & 0.533 \\
\hline Rural resident & $0^{\mathrm{a}}$ & - & - & - & - \\
\hline Student & 33.117 & 0.045 & $2.4 \times 10^{14}$ & 2.224 & $2.6 \times 10^{27}$ \\
\hline $\begin{array}{l}\text { Gov't } \\
\text { employee }\end{array}$ & -18.193 & 0.997 & $1.3 E-8$ & 0.000 & $-^{c}$ \\
\hline Farmer & 6.366 & 0.369 & 581.795 & 0.001 & $6.2 \times 10^{7}$ \\
\hline Private & $0^{\mathrm{a}}$ & - & - & - & - \\
\hline
\end{tabular}

${ }^{\mathrm{a}}$ Used as a reference for the category. $\mathrm{WBC}=$ White Blood Cell count, $\mathrm{RBC}=$ Red Blood Cell count, and Hgb $=$ Hemoglobin.

$29.6 \%$ was seen among patients who stated they were students; even though being a student does not have any biological relation with leukemia, this may be due to the age distribution that most of the patients in the group $0-14$ years of age are students. Prenatal and early life exposures are thought to be important determinants of childhood leukemia. Several mechanisms by which exogenous and endogenous agents may impact the risk of childhood leukemia have been identified. Exposure to possible carcinogenic or toxic substances early in a female's life may result in permanent damage. Since no new oocytes are formed after birth and their maturation begins during gestation, exposures occurring during this critical time may be of the greatest importance. During pregnancy, exposures to agents such as ionizing radiation may act directly while others may act indirectly via placental transfer. On the other hand, offspring may be subjected postnatally to environmental exposures, both directly and indirectly [31]. As most of the children are from rural areas they may have been exposed to different environmental exposures while they live with their farmer parents.

The types of leukemia were identified by using the FABclassification method, morphological examination stained with Wright's stain, and cytochemical staining with Sudan Black B stain to differentiate between cell lineage. In this study, leukemia was classified as acute leukemia, chronic leukemia, and Myelodysplastic Syndrome, which are then further classified into specific types as acute myeloid leukemia (AML), Acute Lymphoid Leukemia (ALL), Chronic Myeloid Leukemia (CML), Chronic Lymphoid Leukemia (CLL), and Myelodysplastic Syndrome (MDS). The prevalence of acute leukemia was $6.6 \%$, while Chronic Leukemia and Myelodysplastic Syndrome account for $2.4 \%$ and $0.3 \%$, respectively. Out of the total leukemia cases, the proportion of acute and chronic leukemia showed $70.96 \%$ and $25.80 \%$, respectively. This was in line with the finding from Nepal and Pakistan $[1,32]$, while it was in contrast with a study from Albania [33].

Regarding the distribution of some hematological parameters with types of leukemia. In acute leukemia, neutropenia (60.0\%) and thrombocytopenia (76.2\%) were found, while in chronic leukemia, Neutrophilia (71.4\%), Basophil leukocytosis (100.0\%), and eosinophilia (85.7\%) were observed. This result is supported by the biological process that in acute leukemia, due to the infiltration of malignant immature cells in the bone marrow and maturation arrest, there will be neutropenia, anemia, and thrombocytopenia in the peripheral circulation [34, 35]. On 
the other hand, chronic leukemia is characterized by markedly increased bone marrow activity, resulting in an increased number of white blood cells in the bone marrow as well as in the circulation, and for CLL in the lymph nodes. In CML, morphologically and functionally normal granulocytes, predominantly comprised of neutrophils and myelocytes are present. Basophil leukocytosis is a distinct feature of CML, although eosinophilia also usually present [36].

Of the types, the most frequent one was AML, 38.7\% $(n=12)$, whereas the rarely found leukemia type was MDS $(3.2 \%, n=1)$. There was one case that cannot be classified as AML or ALL and grouped as undifferentiated. The rest types of leukemia, ALL, CML, and CLL, have accounted for $29.0 \%$, $19.4 \%$, and $6.5 \%$, respectively. This result was quite different from the American Cancer society's finding which showed that CLL was 32.36\%, CML $14.4 \%$, ALL 9.6\%, from C. S. Hodgson's finding from Canada which rivaled that the highest proportion of leukemia was taken by CLL (44\%), then AML (24\%), CML (12\%), ALL (5\%), and other unspecified subtypes with $15 \%$, and a studyfrom Albania (61.01\% CLL, 10.4\% ALL, 20.21\% AML and 8.3\% CML) $[17,25,32,33]$. On the other hand, it was in line with the report from Nepal [1]. This discrepancy may be due to the difference in demographic structure and low life expectancy in Ethiopia compared to the developed countries [37].

AML was the most common type in both sexes, comprising $66.7 \%$ of cases in men and $33.3 \%$ in women. It was more common in patients older than 50 years (50\%). 77.8\% of ALL was diagnosed before the age of 15 years. CML and CLL were more common in older people-half of CML and all of CLL were diagnosed in those aged 50 and over. This finding was in line with the result from Ireland and Haryana-India $[24,38]$.

Regarding the type of leukemia and associated factors analysis, it was possible only for AML, ALL, and CML because the other types did not fulfill the assumption for multinomial logistic regression. AML was found to be associated with an increase in age $(p=0.019)$, being male in sex $(p=0.03)$, a decrease in RBC count $(p=0.047)$, and rural residency $(p=0.044)$. Individuals who are male, Anemic and rural residents will have a greater odd of being diagnosed with AML as their age increases regardless of their WBC count, hemoglobin level, and platelet count than females with equivalent status.

The association is justified by the fact that when an individual has AML, blasts make copies of themselves quickly, slowing down the production of red blood cells and platelets but increased white blood cell (blasts) in the bone marrow as well as peripheral blood. Also, individuals who live in rural areas are more likely to develop AML due to their lifestyle, which is associated with agricultural activities [29]. On the contrary, the finding of this study did not show a significant relationship with the hemoglobin level and platelet count. But there was a significant mean difference of hemoglobin level $(p<0.001)$ while there was no statistically significant difference of mean on platelet count $(p>0.11)$.

On the other hand, there was no significantly associated variable with the probability of developing ALL or CML. For the rest types of leukemia, CLL, MDS, and undifferentiated leukemia, the regression model was unsuitable to be executed because of the rareness of the cases and the short period of study and hence being unable to fulfill the statistical assumptions.

\section{Conclusions and Recommendations}

From our study, the prevalence of leukemia among patients who have abnormal hematological parameters is significant concerning the GLOBOCAN estimate of leukemia for 2018 in Ethiopia [14]. This reflects that the need for a comprehensive investigation of the general population is required for the planning and management by providing healthcare modality, which helps the diagnosis and effectual treatment of leukemia.

This study showed older age, being male in sex, having anemia, and rural residency of a patient may predict the probability of having acute myeloid leukemia. Thus, laboratory professionals, the first who encounter the patients' results, should perform peripheral morphology assessment as a reflex test for those who have abnormal hematological parameters.

The investigators suggest any other associated factors should be investigated for the complete understating of the distribution and pattern of leukemia in the population. Also, up to date and advanced techniques should be used for better diagnosis, subtype classification, and treatment management of leukemia.

\section{Data Availability}

The SPSS (prevalence of leukemia-full data.sav) data used to support the findings of this study have been deposited in the openICPSR repository (https://doi.org/10.3886/E118941V1).

\section{Conflicts of Interest}

The authors declare that there are no conflicts of interest in this work.

\section{Acknowledgments}

No funding opportunities were obtained for the research process and publication. The authors are grateful to all participants for their willingness to participate and Jimma Medical Center Laboratory staff for their unreserved hands.

\section{References}

[1] R. Kulshrestha and S. P. Sah, "Pattern of occurrence of leukemia at a teaching hospital in eastern region of Nepal-a six year study," vol. 48, no. 173, pp. 35-40, 2009.

[2] D. A. Landau, S. L. Carter, G. Getz, and C. J. Wu, "Clonal evolution in hematological malignancies and therapeutic implications," Leukemia, vol. 28, no. 1, pp. 34-43, 2014.

[3] T. P. Whitehead, C. Metayer, J. L. Wiemels, A. W. Singer, and M. D. Miller, "Childhood leukemia and primary prevention," Current Problems in Pediatric and Adolescent Health Care, vol. 46, no. 10, pp. 317-352, 2016. 
[4] B. Deschler and M. Lübbert, "Acute myeloid leukemia: epidemiology and etiology," Cancer, vol. 107, no. 9, pp. 2099-2107, 2006.

[5] D. Pyatt and S. Hays, "A review of the potential association between childhood leukemia and benzene," Chemico-biological Interactions, vol. 184, no. 1-2, pp. 151-164, 2010.

[6] D. Pyatt, "Benzene and hematopoietic malignancies," Clinics in Occupational and Environmental Medicine, vol. 4, no. 3, pp. 529-555, 2004.

[7] D. P. Sandler, D. L. Shore, J. R. Anderson et al., "Cigarette smoking and risk of acute leukemia: associations with morphology and cytogenetic abnormalities in bone marrow," JNCI Journal of the National Cancer Institute, vol. 85, no. 24, pp. 1994-2003, 1993.

[8] M. Belson, B. Kingsley, and A. Holmes, "Risk factors for acute leukemia in children: a review," Environmental Health Perspectives, vol. 115, no. 1, pp. 138-145, 2007.

[9] J. M. Bennett, D. Catovsky, M.-T. Daniel et al., "Proposals for the classification of the acute leukaemias French-AmericanBritish (FAB) co-operative group," British Journal of Haematology, vol. 33, no. 4, pp. 451-458, 1976.

[10] S. H. Swerdlow, E. Campo, N. L. Harris et al., WHO Classification of Tumours of Haematopoietic and Lymphoid Tissues, IARC, Lyon, France, 2018.

[11] American Cancer Society, Acute Myeloid Leukemia (AML) Subtypes and Prognostic Factors, American Cancer Society Inc., Atlanta, GA, USA, 2018.

[12] C. D. Bloomfield and R. D. Brunning, "The revised FrenchAmerican-British classification of acute myeloid leukemia: is new better?" Annals of Internal Medicine, vol. 103, no. 4, p. 614, 1985.

[13] J. V. Eys, J. Pullen, D. Head et al., "The French-AmericanBritish (FAB) classification of leukemia. The pediatric oncology group experience with lymphocytic leukemia," Cancer, vol. 57, no. 5, pp. 1046-1051, 1986.

[14] M.-F. Adalberto, P. Marion, F. Jacques, S. Isabelle, M. Alain, and B. Freddie, "Epidemiological patterns of leukaemia in 184 countries: a population based study," The Lancet Haematology, vol. 5, no. 1, pp. 14-24, 2018.

[15] Leukaemia Statistics, Australia Cancer Society, 2017, https:// leukaemia.canceraustralia.gov.au/statistics.

[16] K. Seiter, "Acute myeloid leukemia staging: WebMD LLC," 2018, https://emedicine.medscape.com/article/2006750-overview.

[17] American Cancer Society, Cancer Facts and Figures, American Cancer Society Inc., Atlanta, GA, USA, 2017.

[18] M. Solomon Tessema, H. Mahlet Kifle, M. Asefa et al., Estimates of Cancer Incidence in Ethiopia in 2015 Using Population-Based Registry Data, American Society of Clinical Oncology, Alexandria, VA, USA, 2018.

[19] A. M. Zeidan, D. Mahmoud, I. T. Kucmin-Bemelmans et al., "Economic burden associated with acute myeloid leukemia treatment," Expert Review of Hematology, vol. 9, no. 1, pp. 79-89, 2016.

[20] J. Mutuma, J. Wakhungu, and C. Mutai, “The socio economic effect of cancer on patients' livelihoods in Kenyan house holds," Bibechana, vol. 14, p. 37, 2016.

[21] American Cancer Society, The Global Economic Cost of Cancer, American Cancer Society, Atlanta, GA, USA, 2010.

[22] G. Lealem, D. Tesfaye, E. Kaleab et al., "Reference intervals for hematology test parameters from apparently healthy individuals in southwest Ethiopia," SAGE Open Medicine, vol. 6, 2018.
[23] Australian Institute of Health and Welfare, Cancer in Australia (Report No.: Cat. no. CAN 100), Australian Institute of Health and Welfare, Canberra, Australia, 2017.

[24] Ireland National Cancer Registry, Cancer Trends; Leukaemia, National Cancer Registry Ireland, Dublin, Ireland, 2010.

[25] C. S. Hodgson, Blood Cancer in Canada Facts and Statistics, Leukemia and Lymphoma Society of Canada, Toronto, Canada, 2016.

[26] G. Juliusson and R. Hough, "Leukemia," Progress in Tumor Research, vol. 43, pp. 87-100, 2016.

[27] J. Konieczny and L. Arranz, "Updates on old and weary haematopoiesis," International Journal of Molecular Sciences, vol. 19, no. 9, 2018.

[28] F. Ferrara and C. A. Schiffer, "Acute myeloid leukaemia in adults," The Lancet, vol. 381, no. 9865, pp. 484-495, 2013.

[29] P. J. Sinner, J. R. Cerhan, A. R. Folsom, and J. A. Ross, "Positive association of farm or rural residence with acute myeloid leukemia incidence in a cohort of older women," Cancer Epidemiology Biomarkers \& Prevention, vol. 14, no. 10, pp. 2446-2448, 2005.

[30] J. E. Kellerbyrne, S. A. Khuder, and E. A. Schaub, "Metaanalysis of leukemia and farming," Environmental Research, vol. 71, no. 1, pp. 1-10, 1995.

[31] T. Lightfoot, "Aetiology of childhood leukemia," Bioelectromagnetics, vol. 26, no. S7, pp. S5-s11, 2005.

[32] A. R. Khan, M. Ahmad, and S. A. Khan, "Analysis of clinicoepidemiological features of leukemia at a tertiary care facility in Peshawar," International Journal of Medical Sciences, vol. 26, no. 1, pp. 41-45, 2018.

[33] E. Pepa, "The prevalence of leukemia in Albania in the recent year," Interdisplinary Journal of Research and Development, vol. 4, no. 2, 2017.

[34] H. Döhner, D. J. Weisdorf, and C. D. Bloomfield, "Acute myeloid leukemia," New England Journal of Medicine, vol. 373, no. 12, pp. 1136-1152, 2015.

[35] I. D. Kouchkovsky and M. Abdul-Hay, "Acute myeloid leukemia: a comprehensive review and 2016 update," Blood Cancer Journal, vol. 6, no. 7, p. e441, 2016.

[36] A. Ogasawara, H. Matsushita, Y. Tanaka et al., "A simple screening method for the diagnosis of chronic myeloid leukemia using the parameters of a complete blood count and differentials," Clinica Chimica Acta, vol. 489, pp. 249-253, 2019.

[37] The World Bank, Life Expectancy at Birth, Total (Years), World Bank Group, Washington, DC, USA, 2019, https://data.worldbank. org/indicator/SP.DYN.LE00.IN?locations=ET\&view=chart.

[38] R. Radha, V. Minakshi, K. Ashok, and S. Singh, "Incidence of acute and chronic forms of leukemia in Haryana," International Journal of Pharmacy and Pharmaceutical Sciences, vol. 6, no. 2, pp. 323-325, 2014. 